\title{
Disease $X$ und die gefährlichen Gewissheiten moderner Demokratien
}

\author{
Gerrit Tiefenthal ${ }^{1}$
}

\section{Die Pandemie als angekündigte Krise}

Dieser Essay vertritt die These, dass weder der Ausbruch der COVID-19Pandemie noch die Krisenreaktionen westlicher Demokratien auf diese überraschen. Damit ist keinesfalls behauptet, dass es sich um eine invenzione di un'epidemia handelt, wie Giorgio Agamben es im Februar vermutete, war diese doch vielmehr erwartbar als erfunden (vgl. Agamben 2020). Auch wenn die Verbreitungswege und das Ausmaß nicht im Konkreten absehbar waren, war spätestens seit den Epidemien SARS, MERS und der weitaus tödlicheren ,Schweinegrippe' (H1N1) ein Bewusstsein bezüglich der Gefahren viraler Pandemien - besonders in Form von Zoonosen - vorhanden (vgl. Mölling 2020: 17). In diesem Wissen veröffentlichte die WHO 2016 erstmals eine Liste von Erregern, von welchen die vermutlich größten epi- bzw. pandemischen Gefahrenpotentiale für die nächsten Jahrzehnte ausgehen werden. Neben bekannten Krankheiten findet sich dort ein ,Disease $X^{‘}$ genannter Platzhalter. Dieser sollte dabei helfen, sich auf eben solche Pandemien vorzubereiten, deren genaue Parameter nicht vorauszusagen sind: „Disease $X$ represents the knowledge that a serious international epidemic could be caused by a pathogen currently unknown to cause human disease" (WHO 2020). Das mutierte SARS-Virus CoV-2 wurde nach seinem Ausbruch als erster Erreger klassifiziert, der diesen Kriterien einer Disease $X$ entsprach und somit zum ersten Testfall dieses Konzeptes, basierend auf dem Wissen um ein unknown known, Präventionsmaßnahmen einzuleiten. Dass dies offensichtlich nicht in genügendem Maße geschehen ist, lässt sich nicht nur auf die vermeintliche Abstraktheit einer solchen Gefahrenlage zurückführen, vielmehr wurden entsprechende Vorkehrungsmaßnahmen durch gewisse - also auf vermeintlichen Gewissheiten beruhende Grundannahmen westlicher Demokratien behindert. Wenn hier von ,westlichen Demokratien' die Rede ist, dann sind damit die real existierenden

1 Ich danke an dieser Stelle Carolin Schulte für ihre wichtigen und bereichernden Anmerkungen. 
liberal-parlamentarischen Demokratien gemeint, deren oftmals unhinterfragte oder verleugnete Grundlagen es sind, die in Krisenzeiten ihre Gefährlichkeit nicht nur dadurch offenbaren, dass sie Vorkehrungsmaßnahmen verhindern, sondern gleichsam, indem sie gewisse Pfadabhängigkeiten in der Reaktion auf solche Ereignisse als vermeintlich alternativlos erscheinen lassen. Gefährlich werden diese Gewissheiten, da sie zu einem Handeln wider besseren Wissens führen und daher unterstreicht Žižek zurecht, dass in unserer Zeit „die größten Gefahren von de[n] , unbekannt Bekannten' aus[gehen] - d[en] verleugneten Überzeugungen, Vermutungen und obszönen Praktiken, von denen wir vorgeben sie nicht zu kennen, sogar wenn sie die Grundlage unserer öffentlichen Werte sind“ (Žižek 2004). ${ }^{2}$

Krisenzeiten haben das Potential, diesen Selbstbetrug offenzulegen, denn eine Unterbrechung, bspw. in Form eines Ereignisses wie der Coronakrise, öffnet Raum zur Reflexion, zu einem besseren Verstehen dieser problematischen Dispositionen. Wie gefährlich ein solches Handeln wider besseren Wissens sein kann, zeigt sich allein daran, dass die in der ,angekündigten Katastrophe"3 COVID-19-Pandemie auftretenden Probleme weniger externer Natur sind, sondern vielmehr internen Konstellationen und Motiven eines bestimmten Politik- und Gesellschaftsverständnisses folgen. Drei dieser Problemfelder, die in ihrem Wirken als, verleugnete Überzeugungen, Vermutungen und obszöne Praktiken` unmittelbar aufeinander bezogen sind, sollen im Folgenden unter den Begriffen Natürlichkeit, Heiligkeit und Wahrheit genauer betrachtet werden: Erstens (Natürlichkeit) werden animistische Narrative behandelt, die, stets zwischen Allmachtsphantasien und Unterordnungszwängen changierend, gebraucht werden, um vermeintliche Notwendigkeiten - bspw. ökonomischer Natur - zu rechtfertigen. Zweitens (Heiligkeit) wird auf die biopolitischen Dispositionen und Machtzugriffe westlicher Gesellschaften eingegangen, die sich naturgemäß während einer viralen Pandemie deutlich(er) zeigen. Mit Den-

2 Žižek bezieht sich in seinem Text auf Äußerungen, die Donald Rumsfeld vorbrachte, um der Kritik hinsichtlich des Fehlens einer smoking gun, eines Beweises für die Existenz irakischer Massenvernichtungswaffen entgegenzutreten, deren angebliche Existenz von der US-Administration explizit zum casus belli der Invasion von 2003 erhoben wurde. Es sei an dieser Stelle Errol Morris Dokumentarfilm The Unknown Known empfohlen, der sich mit diesem Komplex und besonders der Rolle Rumsfelds beschäftigt. Ferner setzt sich Žižek mit der dahinterstehenden ,Logik' Rumsfelds in seiner Onlinevorlesung The Reality of the Virtual auseinander.

3 So bezeichnete der Medizinhistoriker Philipp Osten die Coronakrise am 24. März in der ORF-Sendung Willkommen Österreich. 
kern des Biopolitischen wie Foucault, Deleuze und Agamben sind moderne westliche Demokratien als Kontrollgesellschaften im ständigen Ausnahmezustand zu verstehen, in denen Vielfalt kontinuierlich unterdrückt, der öffentliche Raum zunehmend depolitisiert, das ohnmächtige (Arendt), heilige, nackte Leben (Agamben) dagegen zunehmend politisiert wird. Denn auch wenn real-existierende Demokratien sich auf den ersten Blick durch eine vermeintlich permanente Erweiterung der Daseinsmöglichkeiten auszeichnen, wird wirkliche Vielfalt in diesen Gesellschaften zumeist positivistischen, rationalistischen und behavioristischen Annahmen geopfert. Drittens (Wabrheit) soll auf die notwendige Demokratisierung von Wissen und Wissenschaft eingegangen werden, die es braucht, um den Gefahren, die sich aus diesen oftmals unhinterfragten Dispositionen westlicher Gesellschaften ergeben, offen zu legen.

\section{Natürlichkeit-Animismen zwischen Notwendigkeit und Allmacht}

Žižek bezeichnete den "triumphant return of capitalist animism" gleich zu Beginn der Pandemie als ein „weird phenomenon" (Žižek 2020a). Seltsam ist nicht nur das Phänomen, sondern vielmehr seine Verwunderung, kann doch von einer Rückkehr kapitalistischer Animismen in Zeiten der Pandemie kaum die Rede sein. Dafür ist man durch die Finanzmarkt- und Währungskrisen der letzten Jahrzehnte zu sehr gewöhnt an Reden, in denen die ,nervösen Märkte' beruhigt und auf ihre natürlichen Gesetzmäßigkeiten vertraut bzw. sich ihnen untergeordnet werden muss. Es scheint genauso zur Normalität geworden zu sein, „social phenomena like markets or financial capital as living entities" zu behandeln, wie eine Abwägung von "thousands who already died (and thousands more who will die)" gegenüber dem ,fact that , markets are getting nervous" ' durchzuführen, ohne ein Bewusstsein der Obszönität solcher Überlegungen erkennen zu lassen (ebd.). Doch ist dies kein Phänomen, das sich erst in den letzten Jahren des beschleunigten Finanzkapitalismus herausgebildet hat. Ein solches Verständnis der Ökonomie als (natur)gesetzmäßig beseeltes Reich der Notwendigkeiten liegt am Grund liberalen ökonomischen Denkens und wird bereits von Marx als Grundproblem bürgerlicher Gesellschaften ausgemacht. Es sind gerade Krisenzeiten und -phänomene, während denen die Virulenz animistischen Denkens deutlich zutage tritt und die es erlauben, diese oftmals unhinterfragten Gewissheiten genauer in Augenschein zu nehmen. Dabei zeigt sich, dass animistische Sichtweisen durch eine „sonderbare Gleichzeitigkeit" (Düttmann 2003: 49) geprägt sind. Auf der einen Seite fördern sie ein Bewusstsein, menschliches Sein und Handeln wirklichen 
wie vermeintlichen Notwendigkeiten zu unterwerfen, auf der anderen implizieren solche Erzählungen die Annahme, dass ein Wissen um die Beseeltheit eines Phänomens gleichsam eine Beherrschung desselben ermögliche, wird doch mit der animistischen Benennung eines Phänomens eine „gewisse Macht über den Träger des Namens gewonnen“ (Freud 2014: 680). Das „animistische System“ ist nicht nur ein Denken von Unterwerfung unter Naturnotwendigkeiten (,ananke ), sondern auch immer „eine Anweisung, wie man verfahren müsse, um der Menschen, Tiere und Dinge, respektive ihrer Geister, Herr zu werden“ (ebd.: 682). Menschen begeistern bzw. beseelen Dinge und Phänomene, um weiterhin einen Einfluss auf diese ausüben zu können. Das dahinterstehende „Prinzip, welches die Magie, die Technik der animistischen Denkweise, regiert, ist das der ,Allmacht der Gedanken““ (ebd.: 688).

Zwar spricht Freud animistisches Denken primär Kulturen im „primitiven" Stadium zu, doch wird diese problematische Fortschrittsidee, die selbst einer beseelten Naturvorstellung im darwinistischen Sinne folgt, an gleicher Stelle wenn nicht revidiert, so doch entschärft - und zwar dort, wo er die „Schicksale“ des Prinzips der „Allmacht der Gedanken“ entlang der drei von ihm angenommenen Phasen der Menschheitsgeschichte, der animistischen, der religiösen und der wissenschaftlichen, erörtert:

„Im animistischen Stadium schreibt der Mensch sich selbst die Allmacht zu; im religiösen hat er sie den Göttern abgetreten, aber nicht ernstlich auf sie verzichtet, denn er behält sich vor, die Götter durch mannigfache Beeinflussungen nach seinen Wünschen zu lenken. In der wissenschaftlichen Weltanschauung ist kein Raum mehr für die Allmacht des Menschen, er hat sich zu seiner Kleinheit bekannt und sich resigniert dem Tode wie allen anderen Notwendigkeiten unterworfen. Aber in dem Vertrauen auf die Macht des Menschengeistes, welcher mit den Gesetzen der Wirklichkeit rechnet, lebt ein Stück des primitiven Allmachtglaubens weiter." (Freud 2004: 691)

Zwar liege der Unterschied zwischen „primitiver“ und „wissenschaftlicher Weltanschauung"vor allem darin, dass erstere eine rein psychologische sei, „sie bedurfte noch keiner Wissenschaft zu ihrer Begründung, denn Wissenschaft setzt erst ein, wenn man eingesehen hat, daß man die Welt nicht kennt und darum nach Wegen suchen muß, um sie kennen zu lernen“ (ebd.: 693). Doch lässt sich das Vertrauen auf des Menschen Geist durchaus als moderner Geisterglaube, die Wissenschaften als moderne Magie bezeichnen. Die sonderbare Gleichzeitigkeit animistischen Denkens findet sich sowohl in den Erzählungen von den nervösen Märkten, wie bei denen der rachsüchtigen und zu bekriegenden Natur(phänomene). Es geht nicht 
nur um eine notwendige oder als notwendig erachtete Unterordnung unter deren Gemütszustände, implizieren solche Annahmen doch gleichsam den Anspruch darauf, dass man wisse, wie man mit diesen umzugehen habe, wie sie zu beruhigen, zu beherrschen seien. Der wissenschaftlichen Moderne ist das Vertrauen in die Allmacht des Menschen nie ganz abhanden gekommen, sondern sie vollzieht eine Geste der gleichzeitigen Beugung vor und Verleugnung der ananke.

So vorsichtig man mit solchen Äußerungen auch sein muss, scheint es, als ließe sich animistisches Denken, die Vorstellung, nicht-menschliche Phänomene seien auf menschenähnliche Weise beseelt, als ideengeschichtliche Konstante bezeichnen, was jedoch keineswegs deterministisch, sondern vielmehr so verstanden werden sollte, dass Animismen Hilfsmittel der Komplexitätsreduktion sind, die es aufgrund der menschlichen Bedingtheit braucht, um sich die Welt zu erschließen. Gleichsam liefern sie in ihrer ,sonderbaren Gleichzeitigkeit ${ }^{`}$ ein gewisses emanzipatorisches Potential in dem Sinne, dass sie ein Gestaltungsvermögen von (Natur-)Phänomenen oder zumindest einen gewissen Einfluss durch menschliches Handeln auf diese implizieren. Akzeptiert man dies, lautet die entscheidende Frage, die es sich in durch die wissenschaftliche Weltanschauung geprägten Demokratien zu stellen gilt, nicht nur, mit welchen Narrativen nach bestem Wissen, gerechnet ${ }^{6}$ werden kann, d.h. es geht um mehr als nur ein Erforschen danach, welche Narrative wirklichen und welche falschen Naturnotwendigkeiten folgen, nämlich immer auch um die Frage, welche gesellschaftlich akzeptabel erscheinen. Dies zeigt sich besonders in Krisenzeiten, wo rasch betäubende (,Die Märkte müssen beruhigt werden!') oder aufrührerische (,Das Virus muss bekämpft werden!') animistische Imperative bei der Hand sind, deren Allmachtsphantasien sich stets in gesellschaftlichen Machtstrukturen und Ordnungsdiskursen widerspiegeln bzw. manifestieren. So können Animismen nicht nur ob ihres reduktionistischen Wesens durchaus problematisch werden, sondern gerade aufgrund ihrer legitimatorischen Funktion. Diese beiden Komplexe werden entlang einer Diskussion der biopolitischen Zwangs- und Ordnungsstrukturen westlicher Gesellschaften, die durchaus durchzogen sind von animistischen Bildern (III.), sowie Möglichkeiten einer Demokratisierung von Wissen und Wissenschaft (IV.), in den nächsten Abschnitten genauer behandelt. 


\section{Heiligkeit - Das (un)politische Subjekt und sein nacktes Leben}

„Und was ist das für eine Gesellschaft, die keinen anderen Wert mehr hat als das eigene Überleben?" fragte Agamben Mitte März in Bezug auf den Umgang mit der Pandemie. Diese Frage enthält den Kern seiner Kritik wider real existierende Demokratien: Die bereitwillige Unterwerfung des (politischen, sozialen, kulturellen etc.) Lebens unter das Paradigma des nackten, bloßen Lebens, unter biopolitische Kontroll- und Überwachungsmechanismen, unter die Logik eines permanenten Ausnahmezustandes. Zwar hat Agamben sich für viele Beobachter*innen durch seine eingangs erwähnte Äußerung, es handele sich um eine erfundene Krise, ins intellektuelle Abseits gestellt und das ominöse Label eines, Verschwörungstheoretikers' eingeheimst, doch bietet sein Werk einen äußerst hilfreichen Fundus an Ansätzen, um die aktuelle Situation, vor allem in ihrer politischen Dimension, zu erfassen. Daher sollten seine Analysen nicht einfach missachtet, sondern durch den Einsatz von Hanlons Rasiermesser („Schreibe nicht der Böswilligkeit zu, was durch Dummheit hinreichend erklärbar ist!“) sauberer konturiert werden. Einer differenzierteren Sichtweise zeigt sich nämlich, dass - sowohl durch Dummheit als auch Böswilligkeit ${ }^{4}-$ nicht nur schon länger der Boden für den Ausbruch der Pandemie, sondern ebenso für die - bisher erfolgten und momentan zur Debatte stehenden - Reaktionsmaßnahmen westlicher Demokratien.

Wie Žižek in Bezug auf Agamben feststellt, wäre es unsinnig, in der Coronapandemie eine Erfindung der herrschenden Klassen zu sehen (vgl. Žižek 2020b). Dafür sei allein der ökonomische Schaden zu groß. Die wirklichen Gefahren bestünden vielmehr darin, sich neuen vermeintlichen Notwendigkeiten unterzuordnen, neue Ordnungs- und Machtzugriffe zu akzeptieren. Dass nämlich eine Verschärfung und Normalisierung dieser Zugriffe in der Vergangenheit oftmals auf Seuchenwellen folgte, ist eine der zentralen Aussagen in Foucaults Überwachen und Strafen. Auf die „Ausschließungsrituale“ der Leprabekämpfung folgten die gesellschaftlichen Ordnungsmodelle der „große[n] Einsperrung“"in Form (absolutistischer)

4 Im arendtschen Sinne verstanden, vgl. Arendt, Hannah: Über das Böse, 2017. Mein Verständnis des Begriffs,Dummheit' folgt an dieser Stelle der Definition von Mukerji und Mannino: „,Dummheit' meint hier nicht etwa einen niedrigen Intelligenzquotienten oder mangelndes Wissen, sondern verbreitete Denkfehler, die in der psychologischen Forschung mittlerweile ausführlich dokumentiert sind. Uns scheint, solche Denkfehler haben Entscheidungsträger in der westlichen Welt im Umgang mit Covid-19 fehlgeleitet. Das ist nur menschlich. Doch ist es deshalb nicht weniger gefährlich.“ (Mukerji/Mannino 2020: 41f.). 
Souveränitätsmacht, „[a]uf die Pest antwortet die Ordnung, die alle Verwirrung zu entwirren hat" mit einer „lückenlos[en] und zentral[en] Registrierung des Pathologischen" und es entwickelten sich die sogenannten „Disziplinierungsgesellschaften“ des 18. Jahrhunderts (Foucault 2016: 253f.). So sei die Dialektik von Freiheit und Disziplinierung der Epoche der Aufklärung unmittelbar eingeschrieben (vgl. ebd.: 285).

Die moderne Epoche der „Verstaatlichung des Biologischen“ in Form von ,Biomacht' folgte nicht unmittelbar einer bestimmten Pandemiewelle, auch wenn, so Foucault, die Pocken und im Besonderen die Cholerawellen des 19. Jahrhunderts entscheidende Faktoren gewesen seien, sondern vielmehr dieser im 18. Jahrhundert beginnenden Bewegung des zunehmend höher werdenden gesellschaftlichen Stellenwerts positivistisch-rationaler Wissenschaften, der ,wissenschaftlichen Weltanschauung', wie Freud sie nennt, und ihrem primären Anliegen, Leben zu erhalten (Foucault 2001: 282). Wie die Disziplinierungsmacht, grenzt sich Biomacht damit von absolutistischer Souveränitätsmacht dahingehend ab, dass den Regierenden zunehmend weniger das Recht zukommt „sterben zu machen“, sondern ihre Macht von nun an „in dem Recht liegt, zugunsten des Lebens zu intervenieren und auf die Art des Lebens und das ,Wie‘ des Lebens einzuwirken“, es „zu verbessern, seine Unfälle, Zufälle, Mangelerscheinungen zu kontrollieren“ (ebd.: 292). Dennoch geht es in der Moderne keineswegs darum, jedes Leben zu schützen. Der höhere Stellenwert des ,Lebens an sich' dient allein der Effizienzsteigerung und Rationalisierung der Bevölkerung. Daher handelt es sich keinesfalls um einen radikalen Bruch zwischen diesen Formen des Zugriffs biopolitischer Macht, sie existieren bis heute nebeneinander.

Dies wird während der Coronapandemie besonders deutlich, in welcher sich nicht nur ein Wiedererstarken disziplinierender, sondern ebenso souveräner Machttechniken beobachten lässt. ${ }^{5}$ Dabei geht es nicht nur um das temporäre Herunterfahren des öffentlichen Lebens, sondern um die Bereitwilligkeit, neue Formen des Überwachens, wie bspw. eine flächendeckende Bewegungsdatenspeicherung, zu akzeptieren. Auf die Gefahren solcher sich intensivierender Maßnahmen hinzuweisen, ist unbedingt notwendig, folgte solchen Erweiterungen der Machtzugriffe historisch doch zumeist eine Depolitisierung von Entscheidungsprozessen und des öffentlichen Raumes, die auch nach Beendigung der Krisensituation nicht wieder revidiert wurden.

5 Vgl. diesbezüglich den Text von Simon Duncker Das Corona-Dispositiv in diesem Band. 
Diese Diagnose steht im Zentrum von Agambens Denken, welches Foucaults biopolitische Überlegungen und Arendts Analysen des Totalitarismus „im Fokus des Begriffs vom ,nackten“ oder , heiligen Leben“ konvergieren" lässt (Agamben 2019: 128). Mit der Bezeichnung homo sacer rekurriert Agamben auf eine Figur des antiken römischen Rechts, die man vereinfacht als ,Vogelfreien' bezeichnen könnte, d.i. eine Person, die entrechtet und aus der Gesellschaft verstoßen wurde. Dieser Verstoß ist jedoch kein wirklicher Ausschluss, da sie immer noch unter dem Bann der sie ausschließenden Gemeinschaft steht; nur in einem Verhältnis zu ihr kann sie rechtlos sein. Folgt man Agamben, ist mit Beginn der politischen Moderne genau diese Figur zum grundlegenden Paradigma von (Staats-)Bürgerschaft geworden und zwar in einer zweifachen Bewegung: Sie durchzieht den Einzelnen durch die Politisierung des individuellen zöé, des nackten Lebens „in seiner Namenlosigkeit“, und macht ihn gleichsam zum Glied eines „Volkskörpers“, einer „Bevölkerung“ und so zum „Teil des Politischen" (Agamben 2019: 132f.). Diese Entwicklung bilde „das entscheidende Ereignis der Moderne und markiere gleichsam eine radikale Transformation der klassischen politisch-philosophischen Kategorien" (ebd.: 14). Es handelt sich nicht mehr um ein zoon politikón, verstanden als „lebendes Tier, das auch einer politischen Existenz fähig ist. Der moderne Mensch ist ein Tier, in dessen Politik sein Leben als Lebewesen auf dem Spiel steht" (Foucault 2008: 1137). Mit Arendt sieht Agamben den Grund für den "Niedergang des öffentlichen Raumes“ (Agamben 2019: 13), der gleichsam den „Niedergang der modernen Demokratie“ (ebd.: 20) markiert, in diesem „Vorrang des natürlichen Lebens vor dem politischen Handeln“ begründet (ebd.: 13). Dies zwinge die Demokratie zu einer "geheime[n] Komplizenschaft mit ihrem erbittertsten Feind. [...] [U]nd solange die Widersprüche, die sich daraus ergeben, nicht gelöst sind, werden Nazismus und Faschismus“, die auf ähnliche Weise „die Entscheidung über das nackte Leben zum höchsten Kriterium erhoben haben, bedrohlich aktuell bleiben" (ebd.: 20).

Wie bedrohlich sich diese „innerste Solidarität“ (ebd.: 20), die unheimliche Geschwisterschaft von moderner Demokratie und Totalitarismus in der aktuellen Situation erweisen wird, ist noch nicht in vollem Maße abzusehen. Klar ist jedoch, dass die bisher durchgeführten und - in weit höherem Maße - die angedachten Maßnahmen gegen das Virus, eine weitere Bedrohung für den öffentlichen Raum wie politisches Handeln darstellen können und schlimmstenfalls werden. Die Frage, wer die Souveränität in solchen Entscheidungsprozessen innehat, ist entscheidend dafür, ob der Fortgang nach der Krise eher in eine totalitaristische(re) oder demokratische(re) Richtung führen wird, denn wie es in Überwachen und Strafen 
heißt, begleiteten die Menschen während der Pestwellen stets zwei Träume: einer die Mächtigen und einer die Ohnmächtigen. Wenn in diesem Abschnitt der Blick eher auf den Traum der Mächtigen, auf die Unordnung eine neue, tiefergehende und resistentere Ordnung folgen zu lassen, eingegangen wurde, wird im Folgenden der Blick auf den anderen Traum geworfen, nämlich den, dass auf die Pest eine „Aufhebung der Gesetze und Verbote, [...], der Einsturz der festgelegten und anerkannten Identitäten“ folgen könnte (Foucault 2016: 254). Auch wenn die jetzigen Diskurse zumeist die Hoffnung offenbaren, möglichst rasch wieder zu einem status quo ante zurückzukehren, könnte die Coronakrise doch die Möglichkeit zu einer notwendigen Demokratisierung von Wissen und Wissenschaften bieten, stehen diese Fragen zwar nicht auf den ersten Blick im Zentrum der gesellschaftlichen Debatten der letzten Jahre, liegen sie doch wohl auf deren Grund.

\section{Wahrheit-Expertokratische Diskurse und ihre Gegner}

Habermas sprach im April bezüglich der Coronakrise davon, dass sie uns paradigmatisch zeige, dass wir uns in einer Epoche befänden, in der es „[s]o viel Wissen über unser Nichtwissen und über den Zwang, unter Unsicherheit zu handeln und leben zu müssen“ gebe, wie niemals zuvor (Habermas 2020). Die Bekanntschaft des Unbekannten, das Wissen um das eigene Nichtwissen, das Bewusstsein von Kontingenz und Grundlosigkeit, lassen sich als Signa der Moderne verstehen (vgl. Flügel-Martinsen 2011). Ein Ereignis wie die Pandemie ist ein Prüfstein, an dem sich erweist, wie dem eigenen Selbstverständnis nach ,moderne' Gesellschaften diese Zeichen eines Lebens jenseits von Glauben und Wissen in Krisenzeiten (er)tragen. Und in eben dieser Krise zeigen sich deutlich zwei Abwehrreaktionen gegen die moderne Kondition: auf der einen Seite ein (blinder) Glaube an die Richtigkeit von Expertenwissen und dessen privilegierter Stellung in politischen Entscheidungsprozessen, auf der anderen die komplette Ablehnung desselben. Die bereits seit Längerem schwelenden Konflikte um ,Blasen', ,Fake-News', ,Lügen-“ bzw. ,Lückenpresse' und spaltende Verschwörungsnarrative werden in der aktuellen Situation weiter befeuert. Sicher ist, dass diese Kontroversen durch die zunehmende Etablierung alternativer und ,sozialer ${ }^{6}$ Medien in den letzten Jahrzehnten zugenommen und vielleicht eine historisch neue Intensität erreicht haben. Jedoch handelt es sich dabei keineswegs um ein neues Phänomen, verweisen diese Diskurse doch auf ein Gründungsmotiv abendländischen politischen Denkens: die Gegenüberstellung von Expertenwissen und bloßer „Schein- 
Meinungen der Sterblichen“ (Parmenides), die uns bereits bei Platos Sophistenschelte begegnet.

Grundsätzlich lassen sich zwei Vorgehensweisen festmachen, Expertenwissen in politischen Entscheidungsprozessen einzubinden: „Die eine hält Experten für die endgültige Autorität [...], die andere unterwirft das Expertenurteil einem höheren Gerichtshof" (Feyerabend 1989: 359). Die erste Variante wäre das, was man eine idealtypische Expertokratie nennen müsste. Plato gesteht zwar Expert*innen zu, dass sie einen privilegierten $\mathrm{Zu}$ gang zur Wahrheit hätten und „mit der Wirklichkeit in Verbindung“ stünden, doch tut er dies, weil er sich auf diese Weise „ihren Erfolg erklären kann [...]. Nicht die Experten, sondern Wahrheit und Wirklichkeit sind die eigentlichen Maßstäbe“ und diese werden letzten Endes dem Urteil von „Superexperten“, den Philosophenkönigen, unterworfen (ebd.: 74). Feyerabend hat in seinen Schriften auf das demokratiegefährdende Potential einer unhinterfragten Vorherrschaft von Expertenwissen bzw. den problematischen Wahrheitsansprüchen bestimmter, meist privilegierter Gruppen hingewiesen. ${ }^{6}$ Die Abwertung von Laien sei einer der „grundlegenden Bestandteile der wissenschaftlichen Ideologie“, nach der allein „Wissenschaftler verstehen können, was ein Wissenschaftler im Sinn hatte, und nur ein Wissenschaftler [...] entscheiden kann, wie ein Wissenschaftler eingesetzt werden soll“ (Feyerabend 1996: 47). In der Nachfolge eines der prominentesten antiken Antagonisten dieser „Expertenideologie“ befürwortet Feyerabend eine stete direkt-demokratische Kontrolle von Expert*innen und ihren Ressentiments:

„Nach Protagoras entdecken die Bürger einer freien Gesellschaft, in der die Information ungehindert nach allen Richtungen fließt, bald die Stärken und die Schwächen ihrer Fachleute. Wie die Geschworenen bei einer Geschworenenverhandlung erkennen sie, daß die Fachleute die Wichtigkeit ihrer Arbeit übertreiben: daß verschiedene Fachleute oft zu verschiedenen Ansichten kommen; daß sie gut informiert sind auf diesem engen Gebiet, aber ausserhalb dieses Gebiets einfach nichts wissen [...]; daß sie vorgeben, nach der Wahrheit zu suchen und sich an die Vernunft zu halten, wenn Ruhm und nicht Wahrheit, Vorurteile, nicht die Vernunft die Leitsterne sind.“ (Feyerabend 2016: 138)

6 Der Ansatz Feyerabends, auf die moderne Kondition eines Lebens jenseits aller Gewissheiten mit einem Mehr an Demokratie zu Antworten, steht in deutlicher Nähe zu Überlegungen, die sich in Frankreich bei Lefort oder Rancière finden. 
In diesem Sinne erscheint eine Relativierung und Kontrolle von Expertenwissen und -wahrheiten in politischen Entscheidungsprozessen, gerade in Krisenzeiten, nicht nur aus demokratietheoretischen Erwägungen angebracht, sondern verspricht auch resistenter und nachhaltiger zu sein als der Ausschluss ,bloßer Laienmeinungen', blindes Vertrauen oder gar bequeme Unmündigkeit. Denn wie bereits im Kontext der biopolitischen Dispositionen moderner Demokratien erläutert, birgt die wissenschaftliche Weltanschauung, „dasselbe Unternehmen, das einst den Menschen die Kraft gab, sich von Ängsten und Vorurteilen einer tyrannischen Religion zu befreien“, die Gefahr, die Menschen „nun zu Sklaven seiner eigenen Interessen“ zu machen (Feyerabend 1980: 122). Das heißt jedoch keinesfalls darauf abzuzielen, Wissenschaften und Wissenschaftler*innen generell aus politischen Entscheidungsprozessen zu entfernen. Es geht um eine interne und externe Demokratisierung der Wissenschaften, die - und das gilt es sich immer bewusst zu machen - keineswegs ,die begriffliche Einheit [bilden], die man ihnen oft auferlegt, noch ist die Vielfalt der Praktiken, aus denen sich das Gebiet der Wissenschaft zusammensetzt, so scharf von anderen Bereichen getrennt" (Feyerabend 2016: 8). ${ }^{7}$

Im bisherigen Umgang mit der Coronakrise wurde Expertenwissen zwar stets dem Urteil eines ,höheren Gerichtshofes` unterworfen, nur fanden diese Prozesse hinter verschlossenen Türen statt und der Gerichtshof wurde keineswegs besetzt durch Bürger*innen, sondern durch eine Klasse politischer Superexpert*innen. ,Die Bevölkerung' wurde lediglich - mal mehr, mal weniger ausführlich begründet - über die Ergebnisse informiert. Dass einige ,Verschwörungstheoretiker*innen' die Notwendigkeiten, die mit einer viralen Pandemie einhergehen, nicht akzeptieren wollen oder gar deren Existenz bestreiten, liegt nicht allein daran, dass sie einem ,primitiven' Stadium reflexiven Denkens verhaftet sind, sondern resultiert vielfach aus einem Misstrauen gegenüber ,der Politik', begründet in intransparenten Entscheidungsprozessen wie diesen. Wenn Demokratien ihrem Selbstanspruch folgen wollen, dann muss selbst auf vermeintlich anti-wissenschaftliche Stimmen nicht mit Ausschluss aus dem Diskurs, sondern mit einem Mehr an Demokratie geantwortet werden. Es braucht eine generelle Demokratisierung von Entscheidungsprozessen, selbst auf die potentielle Gefahr hin, dass dies - wie auch immer geartete - ,Erfolgsaussichten' gefährden sollte. Wie groß die Gefahr ist, dass faschistoide Gruppen sich ansonsten mit Erfolg einer demokratischen Scheinrhetorik bedie-

7 Siehe auch den Beitrag von Oliver Flügel-Martinsen Zeit der Pandemie, Zeit der harten Wissenschaften? in diesem Band. 
nen, sollte nicht nur mit Blick auf die Bildung einer neuen Querfront, die sich in Kreisen der sogenannten ,Verschwörungstheoretiker"innen' bereits etabliert hat, deutlich werden, sondern auch anhand der Tatsache, dass die AfD den Slogan Mehr Demokratie wagen bereits für sich reklamiert hat (vgl. Flügel-Martinsen 2020: 11).

\section{Anastrophe statt Katastrophe?}

Mit Dieter Claessen lässt sich zwischen einer Kata- und einer Anastophe unterscheiden. Beide Begriffe bezeichnen eine Wende, eine herab (katá) zum Negativen, die andere hinauf (aná) zum Positiven. Will man nicht den Anspruch erheben, eine Kassandra spielen zu wollen, ist es noch nicht möglich abzusehen, welcher dieser Begriffe im Rückblick auf die Coronakrise verwendet werden wird. Žižek hegte bereits zu einem frühen Zeitpunkt der Krise die Hoffnung, dass sich aus ihr eine benötigte Katastrophe (,Sad fact, we need a catastrophe") entwickeln könnte, die mit einer "reinvention of communism" einhergehe (Žižek 2020a). In Anlehnung an den zweiten Teil von Tarantinos Kill Bill vergleicht er die potentielle Wirkung der Krise mit der einer, so obskuren wie fiktiven, Martial Arts-Technik, der Five Point Palm Exploding Heart Technique, deren tödlich verheerende Wirkung nicht unmittelbar, sondern zeitverzögert einsetzt. Es bleibt abzuwarten, ob die Krise wirklich ein Ausmaß annimmt, in dem die Absurditäten und Obszönitäten des Kapitalismus so deutlich zu Tage treten, dass wir in absehbarer Zeit erleben werden, dass er es Bill gleichtut und nach fünf letzten Schritten stirbt. Mit Blick auf die aktuellen Markt- und Börsenentwicklungen scheint sich jedoch abzuzeichnen, dass die ersten Krisengewinner*innen genau die Akteure sind, die recht erfolgreich dabei sind, ein neues „Zeitalter des Überwachungskapitalismus“ (Zuboff) einzuläuten. ${ }^{8}$

Um einen Ausblick darauf zu werfen, wie die Krise möglicherweise doch in eine Anastrophe gewendet werden könnte, soll noch einmal zum Ansatz einer Demokratisierung von Wissen und Wissenschaft zurückgekehrt werden. Wie Alexander Kluge in verschiedenen Äußerungen zur Krise hervorgehoben hat, offenbart diese sowohl die Berechnungsversessenheit unserer Zeit wie deren Schwächen. Zwar ist es sicher rational, auf eine virale Pandemie mit der, lückenlosen und zentralen Registrierung des Pathologischen' zu antworten, doch liegen die Fallstricke in einem solchen

8 Die Anleihen der sogenannten GAFAM-Konzerne (Google, Amazon, Facebook, Apple, Microsoft) stiegen in den ersten Monaten der Krise auf ein Allzeithoch. 
Vorgehen nicht allein darin, dass dieser Antwort potentiell immer totalitaristische Elemente innewohnen, sondern auch darin, dass jede noch so gut berechnete Prognose an die Grenzen der Realität stoßen wird. Das heißt keinesfalls, in der Abwehr des Virus auf solche Hilfsmittel zu verzichten. Es sollte jedoch nicht undiskutiert bleiben, ob ihnen stets der Vorzug vor bspw. poetischen Antworten gewährt werden sollte, bieten diese doch nicht nur das, was man eine ,brauchbare Ergänzung' nennen könnte, sie liefern vielmehr - zumindest die guten unter ihnen - eine lebensweltlichere Perspektive als es naturwissenschaftliche Erzählungen zu tun vermögen. Dass ein solches Bedürfnis durchaus vorherrscht, mag sich allein daran zeigen, dass Camus' Die Pest zu Beginn der Coronakrise bei einigen europäischen Buchhändler*innen zeitweise vergriffen war. Ein ähnliches Phänomen ereignete sich in den USA nach der Wahl Trumps, die Arendts The Origins of Totalitarianism zum Verkaufsschlager werden ließ, ein Werk, das dadurch brilliert, dass es gespickt ist mit Referenzen auf Conrad, Proust, Melville und im Besonderen Kafka, in dessen Werk Arendt ein ideales Beispiel für die emanzipatorische Kraft des Poetischen erkennt, ist es ihm doch immer wieder gelungen, das Lebensgefühl in einer totalitaristischen Atmosphäre bereits vor ihrer vollen Ausbildung literarisch zu antizipieren.

\section{Literaturverzeichnis}

Arendt, Hannah. 2017. Über das Böse. München: Piper.

Agamben, Giorgio. 2019. Homo sacer: Die souveräne Macht und das nackte Leben. Frankfurt/M.: Suhrkamp.

Agamben, Giorgio. 2020. L'invenzione di un'epidemia. https://www.quodlibet.it/gior gio-agamben-l-invenzione-di-un-epidemia. 22.02.2020.

Düttmann, Alexander Garcia. 2003. Odd Moves - Ironische Streifzüge. In: Thorsten Bonacker/André Brodocz (Hrsg.), Die Ironie der Politik: Über die Konstruktion politischer Wirklichkeiten. Frankfurt/M./New York: Campus, 34-51.

Feyerabend, Paul. 1980. Erkenntnis für freie Menschen. Frankfurt/M.: Suhrkamp.

Feyerabend, Paul. 1989. Irrwege der Vernunft. Frankfurt/M.: Suhrkamp.

Feyerabend, Paul. 1996. Experten in einer freien Gesellschaft, In: Thorsten Hinz (Hrsg.), Thesen zum Anarchismus. Berlin: Karin Kramer, 38-57.

Feyerabend, Paul. 2016. Wissenschaft als Kunst. Frankfurt/M.: Suhrkamp.

Flügel-Martinsen, Oliver. 2011. Jenseits von Glauben und Wissen. Philosophischer Versuch über das Leben in der Moderne. Bielefeld: transcript.

Flügel-Martinsen, Oliver. 2020. Radikale Demokratietheorien zur Einführung. Hamburg: Junius.

Foucault, Michel. 2001. In Verteidigung der Gesellschaft. Frankfurt/M.: Suhrkamp. 
Foucault, Michel. 2008. Der Wille zum Wissen. In: Die Hauptwerke. Frankfurt/M.: Suhrkamp, 1021-1150.

Foucault, Michel. 2016. Überwachen und Strafen. Frankfurt/M.: Suhrkamp.

Freud, Sigmund. 2014. Totem und Tabu. In: Gesammelte Werke. Köln: Anaconda, 607-758.

Habermas, Jürgen. 2020. So viel Wissen über unser Nichtwissen gab es noch nie. https:// www.fr.de/kultur/gesellschaft/juergen-habermas-coronavirus-krise-covid19-inter view-13642491.html. 10.4.2020.

Mölling, Karin. 2020. Viren - Supermacht des Lebens. München: C.H. Beck.

Mukerji, Nikil/Mannino, Adriano. 2020. Covid-19: Was in der Krise zählt. Über Philosophie in Echtzeit. Stuttgart: Reclam.

World Health Organization. 2020. Prioritizing diseases for research and development in emergency contexts. https://www.who.int/activities/prioritizing-diseases-for-researc h-and-development-in-emergency-contexts.

Žižek, Slavoj. 2004. What Rumsfeld doesn't know about Abu Ghraib. https://www.laca n.com/zizekrumsfeld.htm. 21.5.2004.

Žižek, Slavoj. 2020a. Coronavirus is, Kill Bill'-esque blow to capitalism and could lead to reinvention of communism. https://www.rt.com/op-ed/481831-coronavirus-killbill-capitalism-communism/. 27.02.2020.

Žižek, Slavoj. 2020b. Monitor and Punish? Yes, Please! https://thephilosophicalsalon. com/monitor-and-punish-yes-please/. 16.03.2020. 